\title{
Secondary bacterial infections complicating skin lesions
}

\author{
ITZHAK BROOK \\ Department of Pediatrics, Georgetown University School of Medicine, Washington, DC, USA
}

\begin{abstract}
Secondary bacterial infection in skin lesions is a common problem. This review summarises a series of studies of the microbiology of several of these infections: scabies, psoriasis, poison ivy, atopic dermatitis, eczema herpeticum and kerion. Staphylococcus aureus and group $A$-haemolytic streptococci were the most prevalent aerobes and were isolated from all body sites. In contrast, organisms that reside in the mucous membranes close to the lesions predominated in infections next to these membranes. In this fashion, enteric gram-negative bacilli and Bacteroides spp. were found most often in buttock and leg lesions. The probable sources of these organisms are the rectum and vagina, where they normally reside. Group A $\beta$-haemolytic streptococci, pigmented Prevotella and Porphyromonas spp. and Fusobacterium spp. were most commonly found in lesions of the head, face, neck and fingers. These organisms probably reached these sites from the oral cavity, where they are part of the normal flora. This review highlights the polymicrobial aerobic-anaerobic microbiology of secondarily infected skin lesions.
\end{abstract}

\section{Introduction}

Skin and soft-tissue infections are among the most common infections, and may lead to serious local and systemic complications. These infections can be potentially life-threatening and may progress rapidly; therefore, their early recognition and proper medical and surgical management are important. One of the common causes of skin and soft-tissue infections is the occurrence of secondary bacterial infection that complicates skin lesions. The skin lesions that can be secondarily infected with bacteria are scabies [1], psoriasis [2], poison ivy [3], atopic dermatitis [4], eczema herpeticum [5] and kerion [6].

Many wound and skin infections that complicate skin lesions are caused by mixed bacterial flora. Aerobic and anaerobic, gram-negative and gram-positive organisms, whose origins are the endogenous oral, gastrointestinal and skin flora may be present in such infections, where they exist synergically.

This review summarises a series of studies of the microbiology of secondary bacterial infections compli-

Received 22 March 2002; accepted 2 May 2002.

Corresponding author: Professor I. Brook, 4431 Albemarle St NW. Washington DC 20016, USA. (e-mail: ib6@ georgetown.edu). cating skin lesions. They were retrospective studies in which the clinical and microbiology laboratory records of patients whose specimens of infected sites were processed for the presence of aerobic and anaerobic bacteria were reviewed.

\section{Scabies}

Aerobic and anaerobic bacteria were grown from specimens obtained from 30 children with secondarily infected scabies lesions [1] (Table 1). Aerobic or facultative bacteria only were present in 14 (47\%) patients, anaerobic bacteria only were present in six (20\%) patients, and a mixed anaerobic-aerobic flora was present in $10(33 \%)$ patients. Fifty isolates were obtained from the 30 cases ( 1.7 per specimen); 27 were aerobic or facultative bacteria and 23 were strict anaerobes. The predominant aerobic and facultative bacteria were Staphylococcus aureus (9 isolates), group A streptococci (5), and Pseudomonas aeruginosa (3). The predominant anaerobes were Peptostreptococcus spp. (9) and pigmented Prevotella and Porphyromonas spp. (4). There were single bacterial isolates from nine $(30 \%)$ patients; five of these were $S$. aureus. Sixteen isolates from $12(40 \%)$ patients produced $\beta$-lactamase. Micro-organisms that resided in the mucous membranes close to or in contact with the lesions predominated in those infections. Enteric gram-negative bacilli were isolated from leg and trunk lesions. Group 
Table 1. Micro-organisms isolated from 150 secondarily infected skin lesions

\begin{tabular}{|c|c|c|c|c|c|c|c|c|c|c|c|c|c|c|c|c|c|c|c|c|c|c|c|c|c|c|c|c|c|c|c|}
\hline \multirow[b]{2}{*}{ Isolates } & \multicolumn{4}{|c|}{ Finger } & \multicolumn{5}{|c|}{ Hand } & \multicolumn{5}{|c|}{ Leg } & \multicolumn{5}{|c|}{ Trunk } & \multicolumn{4}{|c|}{ Face and Neck } & \multicolumn{3}{|c|}{ Buttocks } & \multicolumn{5}{|c|}{ Total } \\
\hline & $\mathrm{S}$ & PI & $\mathrm{E}$ & $\mathrm{EH}$ & $\mathrm{S}$ & $\mathrm{P}$ & PI & $\mathrm{E}$ & $\mathrm{EH}$ & $\mathrm{S}$ & $\mathrm{P}$ & PI & $\mathrm{E}$ & EH & $\mathrm{S}$ & $\mathrm{P}$ & PI & $\mathrm{E}$ & $\mathrm{EH}$ & $\mathrm{P}$ & PI & $\mathrm{E}^{*}$ & EH & $\mathrm{P}$ & PI & E & $\mathrm{S}$ & $\mathrm{P}$ & PI & $\mathrm{E}$ & $\mathrm{EH}$ \\
\hline $\mathrm{n}$ & 13 & 5 & 6 & 6 & 6 & 7 & 8 & 6 & 4 & 6 & 8 & 9 & 6 & 3 & 5 & 3 & 4 & 5 & 5 & 2 & 5 & 13 & 5 & 3 & 2 & 5 & 30 & 23 & 33 & 41 & 23 \\
\hline \multicolumn{32}{|l|}{ Aerobic bacteria } \\
\hline S. aureus & 6 & 3 & 3 & 2 & 2 & 4 & 4 & 3 & 1 & 1 & & 3 & 2 & 1 & & 3 & 2 & 3 & 2 & 1 & 1 & 1 & 2 & 2 & & & 9 & 15 & 13 & 12 & 8 \\
\hline S. epidermidis & 2 & & 1 & 1 & & 1 & & & & & & & & & & 1 & & & & & 1 & 1 & & & & & 2 & 2 & 1 & 2 & 1 \\
\hline Streptococcus sp. & 2 & & 2 & & & & & & & & & & & & & & & & 1 & & & & & & & & 2 & & & 2 & 1 \\
\hline Group A Streptococcus & 3 & 1 & 2 & 3 & 2 & 1 & 2 & 1 & 1 & & & 1 & 1 & & & & & & 1 & & 2 & 1 & 1 & & & & 5 & 1 & 6 & 5 & 6 \\
\hline Group D Enterococcus & & & & & & & & & & 1 & & 1 & 1 & & & & & & & & & & & 1 & & & 1 & 2 & 1 & 1 & \\
\hline E. coli & & & & & & & 1 & & & 2 & & 1 & 2 & 1 & & & & & & & & & & 1 & 1 & 2 & 2 & 2 & 3 & 4 & 1 \\
\hline Enterobacter sp. & & & & & & & & & & 1 & & & & & & & & & & & & & & 1 & 1 & 1 & 1 & 1 & 1 & 2 & \\
\hline P. aeruginosa & & & & & & & & & & 1 & & 1 & 1 & 2 & 2 & & 1 & 1 & 1 & & & & & & & & 3 & & 2 & 2 & 3 \\
\hline K. pneumoniae & & & & & & & & & & & & & & & 1 & & & & & & & 1 & & & & & 1 & & & 1 & \\
\hline Proteus sp. & & & & & & & & & & & & & 1 & & 1 & & & 1 & & & & & & & & 1 & 1 & & & 3 & \\
\hline Subtotal aerobes & 13 & 4 & 8 & 6 & 4 & 6 & 7 & 4 & 2 & 6 & & 7 & 8 & 4 & 4 & 4 & 3 & 5 & 5 & 1 & 4 & 4 & 3 & 5 & 2 & 4 & 27 & 23 & 27 & 34 & 20 \\
\hline Anaerobic bacteria & & & & & & & & & & & & & & & & & & & & & & & & & & & & & & & \\
\hline Peptostreptococcus spp. & 3 & 1 & 3 & 1 & 2 & 2 & 2 & 2 & 2 & 1 & & 1 & 1 & & 3 & & & 3 & 2 & 1 & 2 & 3 & 1 & 1 & 1 & 1 & 9 & 6 & 7 & 13 & 6 \\
\hline Pr. acnes & 2 & & 1 & & 1 & 1 & & & 1 & & & 1 & 1 & 1 & 1 & & 1 & 1 & 1 & & & & & & & & 4 & 2 & 2 & 3 & 3 \\
\hline Clostridium spp. & & & & & & & & & & 2 & & & 2 & & & & & & & & & & & & 1 & 1 & 2 & & 1 & 3 & \\
\hline Eubacterium spp. & & & & & & & & & & & & & & & & & & & & & & 1 & & & & & & & & 1 & \\
\hline Bacteroides spp. & & & & & & & & & & 2 & & 1 & 1 & 1 & & & & & & & & & & 1 & 1 & 2 & 2 & 2 & 2 & 3 & 1 \\
\hline $\begin{array}{l}\text { Pigmented Prevotella \& } \\
\text { Porphyromonas spp. }\end{array}$ & 4 & 2 & 3 & 2 & & 2 & & & & & & & & & & & & & & & 2 & 5 & 2 & & & & 4 & 2 & 4 & 8 & 4 \\
\hline Fusobacterium spp. & 2 & & 1 & 1 & & 1 & & & & & & & & & & & & & & & 2 & 3 & 1 & & & & 2 & 1 & 2 & 4 & 2 \\
\hline Subtotal anaerobes & 11 & 3 & 8 & 4 & 3 & 6 & 2 & 2 & 3 & 5 & 3 & & 5 & 2 & 4 & 0 & 1 & 4 & 3 & 1 & 6 & 12 & 4 & 2 & 3 & 4 & 23 & 13 & 18 & 35 & 16 \\
\hline Candida spp. & & & 1 & 1 & & & & & & & & & & & & & & & & & & & 1 & & & 2 & & & & 3 & 2 \\
\hline Total & 24 & 7 & 17 & 11 & 7 & 12 & 9 & 6 & 5 & 11 & & 10 & 13 & 6 & 8 & 4 & 4 & 9 & 8 & 2 & 10 & 16 & 8 & 7 & 5 & 10 & 50 & 36 & 45 & 72 & 38 \\
\hline
\end{tabular}

S, scabies; P, psoriasis; PI, poison ivy; E, eczema; EH, eczema herpeticum.

*Also includes scalp. 
A $\beta$-haemolytic streptococci and $S$. aureus predominated in finger and hand lesions. Bacteroides spp. and Clostridium spp. were isolated from leg lesions, and pigmented Prevotella spp. and Porphyromonas and Fusobacterium spp. were isolated from finger lesions.

\section{Psoriasis}

Bacterial growth was noted in 23 specimens from secondarily infected psoriasis lesions [2] (Table 1). Aerobic or facultative bacteria only were present in 12 $(52 \%)$ patients, anaerobic bacteria only in four $(17 \%)$ and mixed anaerobic-aerobic flora was present in seven (30\%) (Table 1). Thirty-six isolates were obtained from the 23 specimens (1.6 per specimen); 23 aerobic or facultative bacteria and 13 strict anaerobes. The predominant aerobic and facultative bacteria were $S$. aureus (15 isolates), group D Enterococcus (2) and Escherichia coli (2). The predominant anaerobes were Peptostreptococcus spp. (6 isolates) and Bacteroides spp., Propionibacterium acnes and pigmented Prevotella spp. in two each. Single bacterial isolates were obtained from $14(61 \%)$ patients, 11 of which were $S$. aureus. Nineteen of the micro-organisms isolated from $18(78 \%)$ patients produced $\beta$-lactamase. S. aureus was isolated from all body sites. Micro-organisms that resided in the mucous membranes close to the lesions predominated in these infections. Enteric gram-negative rods and Bacteroides spp. predominated in lesions on the legs and buttocks. Group A $\beta$-haemolytic streptococci, pigmented Prevotella spp. and Fusobacterium spp. were isolated most frequently from lesions of the hand.

\section{Poison-ivy dermatitis}

Bacterial growth was noted in 33 specimens from patients with secondarily infected poison-ivy dermatitis [3] (Table 1). Aerobic or facultative bacteria only were present in $18(55 \%)$ patients, anaerobic bacteria only in seven $(21 \%)$ and mixed anaerobic-aerobic bacteria in eight (24\%) (Table 1). Forty-five isolates were obtained from 33 specimens (1.4 per specimen); 27 aerobic or facultative bacteria, and 18 strict anaerobes. The predominant aerobic and facultative bacteria were $S$. aureus (13 isolates) and group A $\beta$-haemolytic streptococci (6). The predominant anaerobes were Peptostreptococcus spp. (7 isolates), pigmented Prevotella and Porphyromonas spp. (4), and Fusobacterium spp. (2). Single bacterial isolates were obtained from 18 (55\%) patients, eight of which were $S$. aureus. Nineteen of the micro-organisms isolated from $16(48 \%)$ patients produced $\beta$-lactamase. Bacteria that resided in the mucous membranes close to the lesions predominated in those infections. Enteric gram-negative rods and Bacteroides spp. predominated in leg and buttock lesions. Group A $\beta$-haemolytic streptococci, pigmented Prevotella and Porphyromonas spp. and Fusobacterium spp. were isolated most frequently from lesions of the finger, face and neck.

\section{Atopic dermatitis}

Bacterial growth was noted in 41 specimens from patients with secondarily infected atopic dermatitis lesions [4] (Table 1). Aerobic or facultative bacteria only were present in $15(36 \%)$ patients, anaerobic bacteria only in eight (20\%) and mixed anaerobicaerobic flora was present in 18 (44\%) (Table 1). Seventy-two isolates were obtained from 41 specimens (1.8 per specimen); 34 aerobic or facultative bacteria, 35 strict anaerobes and three Candida spp. The predominant aerobic and facultative bacteria were $S$. aureus (12 isolates), group A $\beta$-haemolytic streptococci (5) and E. coli (4). The predominant anaerobes were Peptostreptococcus spp. (13 isolates), pigmented Prevotella and Porphyromonas spp. (8) and Fusobacterium spp. (4). Single bacterial isolates were obtained from 14 (34\%) patients, seven of which were S. aureus. Twenty-one of the micro-organisms isolated from 16 (39\%) patients produced $\beta$-lactamase. Micro-organisms that resided in the mucous membranes close to the lesions predominated in these infections. Enteric gramnegative bacilli and Bacteroides spp. predominated in lesions of the legs and buttocks. Group A $\beta$-haemolytic streptococci, pigmented Prevotella and Porphyromonas spp. and Fusobacterium spp. were isolated most frequently from lesions of the finger, scalp, face and neck.

\section{Eczema herpeticum}

Bacterial growth was present in 23 specimens from patients with eczema herpeticum [5] (Table 1). Aerobic or facultative bacteria only were present in nine (39\%) specimens, anaerobic bacteria only in four $(17 \%)$, mixed aerobic-anaerobic bacteria in eight (34\%) and Candida spp. in two (9\%). A total of 38 isolates (20 aerobes, 16 anaerobes and two Candida spp.) was isolated, an average of 1.7 isolates per specimen (0.9 aerobes, 0.7 anaerobes and 0.1 Candida) (Table 1).

Eight (35\%) lesions yielded a single organism (four were $S$. aureus). S. aureus was present in eight (35\%) infections and was isolated from all areas. S. aureus mixed with anaerobic bacteria was found in two instances, once with Pr. acnes and once with a Peptostreptococcus sp. $S$. aureus was mixed with group A $\beta$-haemolytic streptococci in one case. Group A $\beta$ haemolytic streptococci were isolated mainly from the extremities. Gram-negative aerobes ( $P$. aeruginosa and $E$. coli) were isolated from areas on the leg and trunk.

The predominant anaerobes were Peptostreptococcus spp., which were isolated from all sites. Bacteroides spp. were isolated from the leg and pigmented Prevotella and Porphyromonas spp. as well as Fusobacterium spp. were isolated from the fingers, face and neck. $\beta$ Lactamase activity was detected in 12 isolates from 10 (43\%) patients. 


\section{Kerion}

Tinea capitis can induce a severe inflammatory response with the formation of elevated boggy granulomatous masses called kerions, which often contain pustules. A retrospective study evaluated the fungal and aerobic and anaerobic bacteriology of inflammatory tinea capitis in the form of kerions in the scalp hair in 20 patients [6].

Cultures showed growth of Trichophyton tonsurans in 17 (85\%) patients and Microsporum canis in two $(10 \%), S$. aureus in seven (35\%), gram-negative rods in five $(25 \%)$, Peptostreptococcus spp. in five $(25 \%)$ and pigmented Prevotella spp. in three (15\%) (Table 2). Fungi alone were isolated from five $(25 \%)$ patients and anaerobic bacteria alone from one (5\%). Mixed flora of fungi and aerobic bacteria was found in six $(30 \%)$ instances, fungi and anaerobes in three $(15 \%)$ and fungi with aerobic as well as anaerobic bacteria in five $(25 \%)$. The number of isolates in mixed cultures varied between two and five (average, 3.1 isolates/specimen). The predominant aerobic bacteria were Staphylococcus spp. (10 isolates) and the most commonly isolated anaerobes were Peptostreptococcus spp. (5 isolates) and Prevotella spp. (3 isolates).

This study illustrated that the isolation of potentially virulent aerobic and anaerobic bacteria from kerions is common. It is not clear whether these organisms colonise or infect the kerions; it is possible that bacteria play a secondary role in the inflammatory signs in kerions of some patients.

\section{Discussion}

This review highlights the diversity of the microbiology of secondarily infected skin lesions. However, the relative incidence of individual bacteria in these secondary infections could not be calculated because of the retrospective nature of these studies in that only clinically infected cases were swabbed. S. aureus, the most prevalent aerobe, was isolated from all body sites. Recent data suggest that this organism may induce purulent superinfection as well as enhance the in-

Table 2. Bacteria isolated from 20 kerions

\begin{tabular}{lc}
\hline Micro-organism(s) & $\begin{array}{c}\text { Number }(\%) \\
\text { of isolates }\end{array}$ \\
\hline Aerobic and facultative bacteria & $7(35)$ \\
S. aureus & $3(15)$ \\
Group A $\beta$-haemolytic Streptococcus & $4(20)$ \\
$\alpha$-Haemolytic Streptococcus & $2(10)$ \\
Proteus sp. & $1(5)$ \\
K. pneumoniae & $2(10)$ \\
P. aeruginosa & $5(25)$ \\
Anaerobic bacteria & $2(10)$ \\
Peptostreptococcus spp. & $1(5)$ \\
Propionibacterium spp. & $3(15)$ \\
Eubacterium lentum & 30 \\
Prevotella spp. & \\
Total number of isolates &
\end{tabular}

flammatory process by superantigen-mediated T-cell activation [7].

In contrast, organisms that reside in the mucous membranes close to the lesions predominated in infections next to these membranes. In this fashion, enteric gram-negative bacilli and Bacteroides spp. were found most often in the buttock and leg lesions. The probable sources of these organisms are the rectum and vagina, where they normally reside [8]. Group A $\beta$-haemolytic streptococci, pigmented Prevotella and Porphyromonas spp., and Fusobacterium spp. were most commonly found in lesions of the face, neck and fingers. These organisms probably reached these sites from the oral cavity, where they are part of the normal flora [8]. A similar distribution of bacterial flora was observed in cutaneous abscesses in adults and children $[9,10]$ and in burns in children [11].

The isolation of multiple organisms from more than half of the patients illustrates the polymicrobial nature of secondarily infected skin lesions and the potential for bacterial synergy between the different microbial isolates. Several studies have documented the synergic effect of mixtures of aerobic and anaerobic bacteria in experimental infections $[12,13]$. Various hypotheses have been proposed to explain such microbial synergy. It may be the result of protection from phagocytosis and intracellular killing [14], production of essential growth factors [15] or lowering of oxidation-reduction potentials in host tissue [16].

The data presented demonstrate the presence of $\beta$ lactamase-producing organisms in many secondarily infected skin lesions. These organisms not only survive penicillin therapy but also can protect susceptible bacteria from penicillin by releasing the enzyme into the adjacent tissues or abscess cavity [17].

The effect of local steroid therapy on the initiation and progression of secondary bacterial infection in many secondarily infected skin lesions is uncertain. Steroids may mask inflammation, and may suppress some of the immune response [18].

The exact pathogenic role of the organisms isolated from secondarily infected skin lesions has not yet been determined. The isolation of aerobic and anaerobic organisms - which are isolated less commonly from skin and soft-tissue infections - from infected skin lesions raises questions about their pathogenic role. However, these organisms are known pathogens in certain types of skin and soft-tissue infections. These include diabetic foot infections [19], decubitus ulcers [20] and bite wound infections [21]. Therefore, it is possible that they may have a pathogenic role in infected skin lesions. When cultures are obtained from secondarily infected skin lesions, these should be processed for the isolation of anaerobic as well as aerobic bacteria. 
Although local application of specific therapies (e.g., steroids, anti-scabies insecticides) is the mainstay of therapy, management of secondary bacterial infections is essential. Local application of antibacterial agents and drainage of pus are important components of the treatment of these complications. Treatment of serious skin infection should include systemic antimicrobial therapy. This could be important in the event of poor response to therapy or spread of the infection.

The gram-negative anaerobic bacilli, Prevotella and Fusobacterium spp., previously susceptible to penicillins, have been shown in the past decade to have increased rates of resistance to these and other antimicrobial agents [22]. The production of $\beta$-lactamase is one of the main mechanisms of resistance to penicillins by many gram-negative anaerobic bacilli, including the Bacteroides spp. Complete identification and testing for antimicrobial susceptibility and $\beta$-lactamase production are essential for the management of infections caused by these bacteria.

Antimicrobial therapy for mixed aerobic and anaerobic bacterial infections is required when polymicrobial infection is suspected [23]. Antimicrobial agents that generally provide coverage for $S$. aureus as well as anaerobic bacteria include cefoxitin, clindamycin, carbapenems (i.e., imipenem, meropenem), the combination of a $\beta$-lactamase inhibitor (clavulanic acid) plus a penicillin (e.g., ticarcillin), and the combination of metronidazole plus a $\beta$-lactamase-resistant penicillin. Cefoxitin, the carbapenems and a penicillin plus a $\beta$ lactamase inhibitor also provide cover against members of the family Enterobacteriaceae. However, agents effective against these organisms (i.e., aminoglycosides, fourth-generation cephalosporins and quinolones) should be added to the other agents when treating infections that include these bacteria.

\section{References}

1. Brook I. Microbiology of secondary bacterial infection in scabies lesions. J Clin Microbiol 1995; 33: 2139-2140.
2. Brook I, Frazier EH, Yeager JK. Microbiology of infected pustular psoriasis lesions. Int J Dermatol 1999; 38: 579-581.

3. Brook I, Frazier EH, Yeager JK. Microbiology of infected poison ivy dermatitis. $\mathrm{Br} J$ Dermatol 2000; 142: 943-946.

4. Brook I, Frazier EH, Yeager JK. Microbiology of infected atopic dermatitis. Int J Dermatol 1996; 35: 791-793.

5. Brook I, Frazier EH, Yeager JK. Microbiology of infected eczema herpeticum. J Am Acad Dermatol 1998; 38: 627-629.

6. Brook I, Frazier EH, Yeager JK. Aerobic and anaerobic microbiology of kerions. Pediatr Infect Dis J 1995; 14: 326-327.

7. McFadden JP, Noble WC, Camp RD. Superantigenic exotoxinsecreting potential of staphylococci isolated from atopic eczematous skin. Br J Dermatol 1993; 128: 631-632.

8. Brook I. Indigenous microbial flora of humans. In: Howard RJ, Simmons RL (eds) Surgical infectious diseases, 3rd edn. Norwalk, CT, Appleton \& Lange. 1995: 36-46.

9. Meilslin HW, Lerner SA, Graves MH et al. Cutaneous abscesses: anaerobic and aerobic bacteriology and outpatient management. Ann Intern Med 1977; 87: 145-149.

10. Brook I, Finegold SM. Aerobic and anaerobic bacteriology of cutaneous abscesses in children. Pediatrics 1981; 67: 891-895.

11. Brook I, Randolph JG. Aerobic and anaerobic flora of burns in children. J Trauma 1981; 21: 313-318.

12. Meleney FL. Bacterial synergy in disease processes. With a confirmation of the synergistic bacterial etiology of a certain type of progressive gangrene of the abdominal wall. Ann Surg 1931; 94: 961-981.

13. Brook I, Hunter V, Walker RI. Synergistic effect of Bacteroides, clostridia, fusobacterium, anaerobic cocci, and aerobic bacteria on mortality and induction of subcutaneous abscess in mice. $J$ Infect Dis 1984; 149: 924-928.

14. Ingham HR, Sisson PR, Tharagonnet D, Selkon JB, Codd AA. Inhibition of phagocytosis in vitro by obligate anaerobes. Lancet 1977; 2: 1252-1254.

15. Lev M, Krudell KC, Milford AF. Succinate as a growth factor for Bacteroides melaninogenicus. J Bacteriol 1971; 108: 175-178.

16. Mergenhagen SE, Thonard JC, Scherp HW. Studies on synergistic infections. I: Experimental infections with anaerobic streptococci. J Infect Dis 1958; 103: 33-44.

17. Brook I. The role of beta-lactamase-producing bacteria in the persistence of streptococcal tonsillar infection. Rev Infect Dis 1984; 6: 601-607.

18. Carpenter CB. Immunosuppression in organ transplantation. N Engl J Med 1990; 322: 1224-1226.

19. Sapico FL, Witte JL, Canawati HN, Montgomerie JZ, Bessman AN. The infected foot of the diabetic patient: quantitative microbiology and analysis of clinical features. Rev Infect Dis 1984; 6 Suppl 1: S171-S176.

20. Brook I. Microbiological studies of decubitus ulcers in children. J Pediatr Surg 1991; 26: 207-209.

21. Brook I. Microbiology of human and animal bite wounds in children. Pediatr Infect Dis J 1987; 6: 29-32.

22. Sutter VL, Finegold SM. Susceptibility of anaerobic bacteria to 23 antimicrobial agents. Antimicrob Agents Chemother 1976; 10: $736-752$.

23. Finegold SM. Anaerobic bacteria in human disease. New York, Academic Press. 1977. 